\title{
ESTABILIDAD DURANTE EL ALMACENAMIENTO DE CARNE DE POLLOS ALIMENTADOS CON ORÉGANO SECO (Origanum vulgare L.) EN LA DIETA
}

\section{STORAGE STABILITY OF POULTRY MEAT FROM BROILERS FED WITH DRY OREGANO (Origanum vulgare L.) IN THE DIET}

\author{
Valeria Velasco ${ }^{1 *}$, Paulina Bravo ${ }^{1}$, Pamela Williamss ${ }^{1}$, Jorge Campos ${ }^{1}$, Rita Astudillo ${ }^{1}$, Pedro Melín ${ }^{2}$ \\ ${ }^{1}$ Universidad de Concepción, Departamento de Producción Animal, Facultad de Agronomía, Casilla \\ 537, Chillán, Chile. \\ ${ }^{2}$ Universidad de Concepción, Departamento de Agroindustrias, Facultad de Ingeniería Agrícola, Casilla \\ 537, Chillán, Chile. \\ "Autor para correspondencia E-mail: vvelasco@udec.cl
}

\section{RESUMEN}

Los antioxidantes naturales pueden ser usados para aumentar la estabilidad de la carne de pollo durante el almacenamiento. El objetivo de este estudio fue determinar la estabilidad durante el almacenamiento de carne de pollos alimentados con una dieta suplementada con orégano seco en dosis $0 ; 1,25 ; 2,5 ; 3,75$ y $5 \%$ con respecto a la materia seca ofrecida. Los parámetros evaluados durante el almacenamiento de la carne fueron: índice de peróxidos, recuento de aerobios mesófilos (RAM), color (espacio CIELab), composición nutricional, características sensoriales (color, intensidad de aroma) y aceptación. Se encontró que la carne de pollos alimentados con dietas suplementadas con orégano seco presentó una menor oxidación de lípidos al inicio del almacenamiento en refrigeración, mayores diferencias de color $(\Delta \mathrm{E}>5)$ con respecto al control en los tratamientos con mayor inclusión de orégano $(2,5-5 \%)$ a los siete días de almacenamiento, un RAM sin variación entre tratamientos, un mayor contenido de lípidos en tratamientos con mayor inclusión de orégano ( 3,75 y $5 \%$ ), y una mayor aceptación por el consumidor. Se puede concluir que la estabilidad de la carne de pollos alimentados con orégano seco como ingrediente dietario aumenta al inicio del almacenamiento por la menor oxidación de lípidos.

Palabras clave: antioxidantes, índice de peróxidos, recuento total, espacio CIELab, análisis sensorial.

\section{ABSTRACT}

Natural antioxidants may be used in order to increase storage stability of poultry meat. The aim of this study was to determine the storage stability of poultry meat from broilers fed with diets containing dry oregano at different doses $0,1.25,2.5,3.75$ and $5 \%$. The following parameters were evaluated: peroxides index, total mesophilic aerobic count (RAM), color (CIELab), nutritional composition, sensory characteristics (color and aroma intensity) and consumer acceptance. The results showed that poultry meat from broilers fed with dry oregano exhibited a lower oxidation of lipids at the beginning of refrigerated storage. Regarding color, higher differences $(\Delta E>5)$ were observed in the treatments with higher doses of oregano (2.5-5\%) seven days after storage when compared with the control. No variation in TMA values were found between treatments. Lipid content was higher in the treatments with a higher amount of oregano added (3.75 and $5 \%)$, while consumer acceptance 
was also higher. It can be concluded that the storage stability of poultry meat from broilers fed with diets containing dry oregano increases at the beginning of the storage due to lower lipid oxidation.

Key words: antioxidants, peroxide value, total count, CIELab space, sensory analysis.

\section{INTRODUCCIÓN}

En producción animal los antibióticos han sido ampliamente utilizados con fines terapéuticos, de prevención y de promoción del crecimiento. Sin embargo, debido a la emergencia y propagación de bacterias resistentes a antibióticos, se ha prohibido en algunos países su incorporación en alimentación animal (Huyghebaert et al., 2011), reemplazándose por alternativas no terapéuticas, entre las que se cuenta la inclusión de extractos vegetales, que permiten aumentar la ganancia de peso (Mc Dermott et al., 2002). En producción de pollos de carne no sólo es importante mejorar la eficiencia de la producción, sino también lograr extender la vida útil de la carne, ya que es un producto altamente perecible (Chouliara et al., 2007). La oxidación de los lípidos en la carne de pollo se produce durante el almacenamiento y procesamiento de la carne. La formación de productos secundarios en la oxidación afecta las características sensoriales, como sabor y aroma, lo cual se relaciona con la disminución de su vida útil (Mohamed y Mansour, 2012). En la industria alimentaria se han utilizado antioxidantes sintéticos como butil-hidroxitolueno (BHT), butil-hidroxianisol (BHA) y terbutil-hidroquinona (TBHQ) para retardar la oxidación de lípidos (Fasseas et al., 2007). Sin embargo, existe preocupación por la utilización de estos antioxidantes sintéticos por su riesgo de toxicidad (Okubo et al., 2003; Rodríguez et al 2012).

La carne, además de la oxidación, se puede contaminar con microorganismos que causan enfermedades de transmisión alimentaria (ETAs) durante el procesamiento y almacenamiento en refrigeración, razón por la cual se han buscado alternativas para extender la vida útil de estos productos (Solomakos et al., 2008). Entre estas alternativas se destaca la utilización de productos naturales que inhiben el crecimiento de patógenos (Solomakos et al., 2008; Nieto et al., 2010).

El orégano (Origanum vulgare L.) es una planta aromática que pertenece a la familia Lamiaceae. El aceite esencial (AE) del orégano es conocido por poseer propiedades antimicrobianas (Marino et al., 2001; Friedman et al., 2002), debido a que sus principales componentes son compuestos fenólicos que tienen la capacidad de interactuar con la membrana citoplasmática, provocando la inestabilidad de bacterias y posterior muerte celular (Dorman and Deans, 2000; Lambert et al., 2001). Los principales constituyentes del AE de orégano son el carvacrol, timol, Q-cimeno y Y-terpineno (Bonfanti et al., 2012; De Falco et al., 2014). Se ha demostrado la actividad antimicrobiana del AE de orégano en estudios realizados in vitro contra bacterias Gram-negativas y Gram-positivas, tales como Escherichia coli O157:H7, Listeria monocytogenes, Staphylococcus aureus, Pseudomonas aeruginosa, Bacillus cereus, Bacillus subtilis, Salmonella typhi Ty2, entre otras (Elgayyar et al., 2001; Lambert et al., 2001; Lv et al., 2011; De Falco et al., 2014). Las bacterias Gram-negativas han sido más resistentes a la acción del AE de orégano, debido a la presencia de una membrana externa de lipopolisacáridos que actuaría limitando el acceso de los compuestos activos presentes en el AE (Burt, 2004). Existen estudios que han demostrado la actividad antibacteriana del AE de orégano en carne de pollo durante la refrigeración, obteniendo una reducción del recuento total de microorganismos, y también de Pseudomonas, enterobacterias y bacterias ácido lácticas (Chouliara et al., 2007; Oral et al., 2009).

Los antioxidantes naturales se pueden incorporar en la dieta de los animales, aplicarlos en la superficie de la carne o utilizarlos en envases activos para mejorar la calidad de la carne (Velasco et al., 2010). Se ha logrado extender la vida útil de la carne de pollo durante la refrigeración utilizando AE de orégano en el envasado de la carne (Chouliara et al., 2007; Oral et al., 2009). También existen estudios que han demostrado que el $\mathrm{AE}$ de orégano incorporado en la dieta de animales es capaz de mejorar la estabilidad oxidativa de la carne de ave durante el almacenamiento en refrigeración (Botsoglou et al., 2002; 2003a; 2003b). La suplementación de la dieta de animales con antioxidantes naturales es una estrategia simple y conveniente para incorporar compuestos antioxidantes en la carne, sin la necesidad de tener un etiquetado especial del producto final (Botsoglou et al., 2002).

El objetivo de este estudio fue determinar la estabilidad durante el almacenamiento de carne de pollos alimentados con diferentes dosis de orégano seco en la dieta.

\section{MATERIALES Y MÉTODOS}

\section{Tratamientos}

Se utilizaron 20 pollos de engorda por tratamiento (5 pollos por repetición), híbrido Rock 308 de $45 \mathrm{~g}$ sin sexar y vacunados contra bronquitis aviar, de 1 día de edad. Los animales se alimenta- 
ron durante 42 días en total, tres semanas en etapa de crianza y tres semanas en etapa de engorda. Los niveles de incorporación de orégano seco a la dieta fueron determinados según los resultados obtenidos en otros estudios en corderos (Bampidis et al., 2005) y en pollos (Botsoglou et al., 2002; Botsoglou et al., 2003a). Se utilizó una dieta control y dietas con orégano seco al 1,25; 2,5; 3,75 y $5 \%$, con respecto a la materia seca, con 4 repeticiones cada uno. El orégano, procedente de la Provincia de Nuble, se adquirió en el mercado local.

La formulación de la dieta control se realizó de acuerdo a los requerimientos establecidos por NRC (1994). Se seleccionaron al azar 4 animales del total de 20 pollos por tratamiento (1 pollo por repetición) para realizar los análisis de la carne, obteniendo músculos pectorales y muslos de cada uno. La carne se envasó en bolsas de polietileno, se mantuvo en refrigeración $\left(2^{\circ} \mathrm{C}\right)$ durante una semana después de la faena, y luego en congelación durante 1 mes $\left(-18^{\circ} \mathrm{C}\right)$. Se determinó la composición de la carne de pollo y su aceptación, y durante el almacenamiento se determinó: oxidación de lípidos (índice de peróxidos, IP), recuento de aerobios mesófilos (RAM) y color (espacio CIELab).

\section{Determinaciones}

Índice de peróxidos. Se extrajo una muestra de carne de $150 \mathrm{~g}$ de muslo de cada tratamiento para determinar el índice de peróxidos en duplicado, los día 0 y 7 (refrigeración a $2^{\circ} \mathrm{C}$ ). La determinación se realizó según la metodología descrita por Pearson (1986). Este método consiste en la extracción de lípidos de la carne de pollo mezclada con $250 \mathrm{~mL}$ de cloroformo en agitación por $3 \mathrm{mi}-$ nutos. La solución es filtrada 2 veces con un papel con $1 \mathrm{~g}$ de sulfato sódico anhídrido. Posteriormente, se añade $37 \mathrm{~mL}$ de ácido acético glacial al 100 \% y 1 mL de solución saturada de KI a 25 mL del doble filtrado. Se deja en reposo la solución durante 1 minuto en oscuridad y se agrega $30 \mathrm{~mL}$ de agua destilada, y $1 \mathrm{~mL}$ de almidón de papa (Merck) en $99 \mathrm{~mL}$ de agua, al $1 \%$ peso volumen como indicador y se titula con (solución acuosa) de tiosulfato sódico 0,01 N. El índice de peróxidos (IP) en meq $\mathrm{kg}^{-1}$ se calculó según la ecuación:

$$
I P=\frac{V x C x 100}{m}
$$

dónde $\mathrm{V}$ es el volumen $(\mathrm{mL})$ de tiosulfato sódico; C es la concentración del tiosulfato sódico $(0,01 \mathrm{~N})$ y m es el peso de la muestra ( $\mathrm{kg}$ de grasa).

Recuento de aerobios mesófilos (RAM). Muestras de carne (músculos pectorales y muslos) de cada tratamiento se analizaron los días 0,3 y 7 , durante su refrigeración, según la metodología descrita por Quinn y Markey (2005). Se realizó una homogenización de $10 \mathrm{~g}$ de muestra en $90 \mathrm{~mL}$ de agua peptonada (Merck) y se realizaron diluciones seriadas. Posteriormente, se inocularon placas Petri con medio Plate count y se incubaron en condiciones de aerobiosis a $37^{\circ} \mathrm{C}$ por 24 horas. Finalmente, se realizó el recuento de colonias de microorganismos mesófilos aerobios.

Determinación de color. Se midió en carne de músculos pectorales y muslos en 4 muestras de cada tratamiento, en triplicado, los día 0,3 y 7 durante su refrigeración. Se utilizó un colorímetro de reflectancia (Hunter Lab 45/0 Color Quest, Hunter Associates Laboratory Inc., Reston, Virginia, USA) que mide las coordenadas de color del espacio CIELab, donde, $a^{*}$ mide la intensidad de rojo desde $a+$ (rojo) hasta $a-$ (verde), $b^{*}$ mide la intensidad del amarillo desde $\mathrm{b}+$ (amarillo) hasta b- (azul) y L* indica luminosidad de 0 (negro) a 100 (blanco). Se determinó el croma $\left(C^{*}\right)$, el ángulo de tono $\left(\mathrm{h}^{*}\right)$ y la diferencia de color $(\Delta \mathrm{E})$ de la carne con el control, a través de las siguientes ecuaciones (Hunter Associates Laboratory, 2008)

$$
\begin{aligned}
& C^{*}=\sqrt{\left(a^{* 2}+b^{* 2}\right)}(2), \\
& h=\arctan \left(\frac{b^{*}}{a^{*}}\right) \\
& \Delta E=\sqrt{\left(L_{1}^{*}-L_{2}^{*}\right)^{2}+\left(a_{1}^{*}-a_{2}^{*}\right)^{2}+\left(b_{1}^{*}-b_{2}^{*}\right)^{2}}
\end{aligned}
$$

Análisis composición nutricional. Para caracterizar la carne de pollo se realizó un análisis proximal, para lo cual se extrajo $100 \mathrm{~g}$ de músculos pectorales y $100 \mathrm{~g}$ de muslos por separado de cada tratamiento, en duplicado, utilizando los siguientes métodos: Humedad: mediante secado en liofilizador (Christ Alpha 1-4 LD Plus, Osterode, Germany) a $-65^{\circ} \mathrm{C}$ por $48 \mathrm{~h}$. Proteínas: por el método de Kjeldhal, según el método 981.10 (AOAC, 1997). Lípidos: determinado como extracto etéreo, mediante el método 960.39 (AOAC, 1997). Minerales: a través de la determinación de cenizas, según el método 920.153 (AOAC, 1997).

Evaluación sensorial. Se conformó un panel de 16 degustadores, de los cuales 9 tenían categoría de "especializados", pertenecientes a una empresa productora de carne de aves, y 7 con categoría "no especializados". La muestras de carne de músculo pectoral de pollo congeladas a $-18^{\circ} \mathrm{C}(1$ mes después de la faena) se descongelaron ( $24 \mathrm{~h}$ a $2^{\circ} \mathrm{C}$ ) y se cortaron en trozos de $2,0 \times 3,0 \mathrm{~cm}$, con un peso entre 6,5 a $7,5 \mathrm{~g}$. Luego, se sometieron a cocción en un horno eléctrico a $190^{\circ} \mathrm{C}$ por 15 minutos aproximadamente, hasta que la temperatura interna alcanzó $75^{\circ} \mathrm{C}$. Posteriormente, las muestras se depositaron sobre recipientes blan- 
cos, identificadas con un código de 3 dígitos al azar. Cada panelista evaluó una muestra de cada tratamiento, utilizando agua entre cada muestra. Se evaluaron las características sensoriales de color, aroma y aceptación, mediante una escala de evaluación (Tabla 1) (Wittig de Penna, 2001).

\section{Diseño experimental y análisis estadístico}

El diseño experimental fue completamente al azar con cinco tratamientos y cuatro repeticiones para cada tratamiento. Los datos fueron sometidos a análisis de varianza al 5\% de significancia. En el caso de presentarse diferencias significativas, los datos fueron sometidos al test de contraste de Duncan al 5\% de significancia. Los supuestos del análisis de varianza fueron verificados a través de los test de Shaphiro - Wilks modificado para la normalidad y Bartlet para la homogeneidad de varianza, ambos al 5\% de significancia. Para el análisis sensorial se utilizó un diseño completamente al azar con 16 repeticiones, donde cada juez fue una repetición. Tanto para la evaluación sensorial como para el recuento de aerobios mesófilos se utilizó el análisis de varianza no paramétrico de Kruskal - Wallis al 5\% de significancia y como test de contraste se utilizó el propuesto por Conover al 5\% de significancia. El software que se utilizó fue InfoStat professional versión 2008 (Balzarini et al., 2008).

\section{RESULTADOS Y DISCUSIÓN}

\section{Oxidación de lípidos}

La lipoperoxidación o enranciamiento ocurre por acción de sustancias denominadas radicales libres (RL) que poseen uno o más electrones desapareados. Los RL sustraen un hidrógeno de un ácido graso, generalmente poliinsaturado que posee múltiples dobles enlaces, entre los cuales existen grupos metileno $\left(\mathrm{CH}_{2}\right)$, cuyos hidrógenos son reactivos. De esta forma el RL se estabiliza y el ácido graso se convierte en un radical lipídico (L), el cual en presencia de oxígeno se transforma en un radical lipoperóxido. Este radical lipoperóxido buscará estabilizarse sustrayendo un $\mathrm{H}$ a un ácido graso vecino transformándose de esta manera en un lipohidroperóxido (LOOH). Todo lo anterior genera una reacción en cadena, denominada lipoperoxidativa, que produce la oxidación progresiva de los lípidos presentes en la carne (Richards et al., 2002, Chaijan, 2008).

La influencia de la suplementación con orégano seco en la oxidación de lípidos durante el almacenamiento de carne de muslo de pollo se puede observar en la Fig. 1. Los análisis de oxidación de lípidos se realizaron en el muslo, debido a que el contenido de grasa es mayor en este corte que en el músculo pectoral (Hashim et al., 2013). Al inicio del estudio (día 0) la carne presentó mayor oxidación de lípidos que en el día 7 , ya que los $\mathrm{LOOH}$ formados inicialmente son descompuestos en aldehídos y otras sustancias de descomposición de las grasas, las cuales se acumulan afectando las características organolépticas (Richards et al., 2002; Chaijan, 2008). El tratamiento sin adición de orégano en la dieta $(\mathrm{C})$ presentó un mayor índice de peróxidos en el día 0 que los demás tratamientos, disminuyendo el índice de peróxidos en la medida que aumenta la inclusión de orégano en la dieta de los pollos. Así, los tratamientos de inclusión de orégano seco en la dieta de 3,75\% y $5 \%$ presentaron un menor índice de peróxidos inicial comparado con los otros tratamientos $(\mathrm{P} \leq$ $0,05)$. No se presentaron diferencias significativas $(P>0,05)$ entre tratamientos en el día 7 . Lo mismo ocurrió en el estudio realizado por Botsoglou et al. (2002), en el que se incluyó AE de orégano a la dieta en dosis de 50 y $100 \mathrm{mg} \mathrm{kg}^{-1}$, obteniendo una respuesta en la capacidad antioxidante en la carne, medida mediante la concentración de malondialdehído durante el almacenamiento en refrigeración, situación que se repite en carne de cordero alimentados con una suplementación de AE de orégano mezclado con el concentrado (1 $\mathrm{mL} \mathrm{kg}^{-1}$ de concentrado) (Simitzis et al., 2008).

\section{Recuento de aerobios mesófilos (RAM)}

El recuento total de aerobios mesófilos durante el almacenamiento de carne de pollos alimentados con dietas con orégano se presenta en la Fig. 2. Se observa que en los días 0 y 3 de almacenamiento en refrigeración el RAM fue menor

Tabla 1. Escalas de evaluación sensorial de carne de músculo pectoral de pollos.

Table 1. Sensory evaluation grades of breast muscle of broilers.

\begin{tabular}{llllll}
\hline & Color & & Intensidad de aroma & & \multicolumn{1}{c}{ Aceptación } \\
\hline 1 & Blanco & 1 & Extremadamente alta & 1 & Me disgusta mucho \\
2 & Blanco-amarillo & 2 & Alta & 2 & Me disgusta \\
3 & Amarillo & 3 & Moderada & 3 & Me es indiferente \\
4 & Pardo & 4 & Baja & 4 & Me gusta \\
& & 5 & No es perceptible & 5 & Me gusta mucho \\
\hline
\end{tabular}

Adaptado de Velasco et al. (2010). 


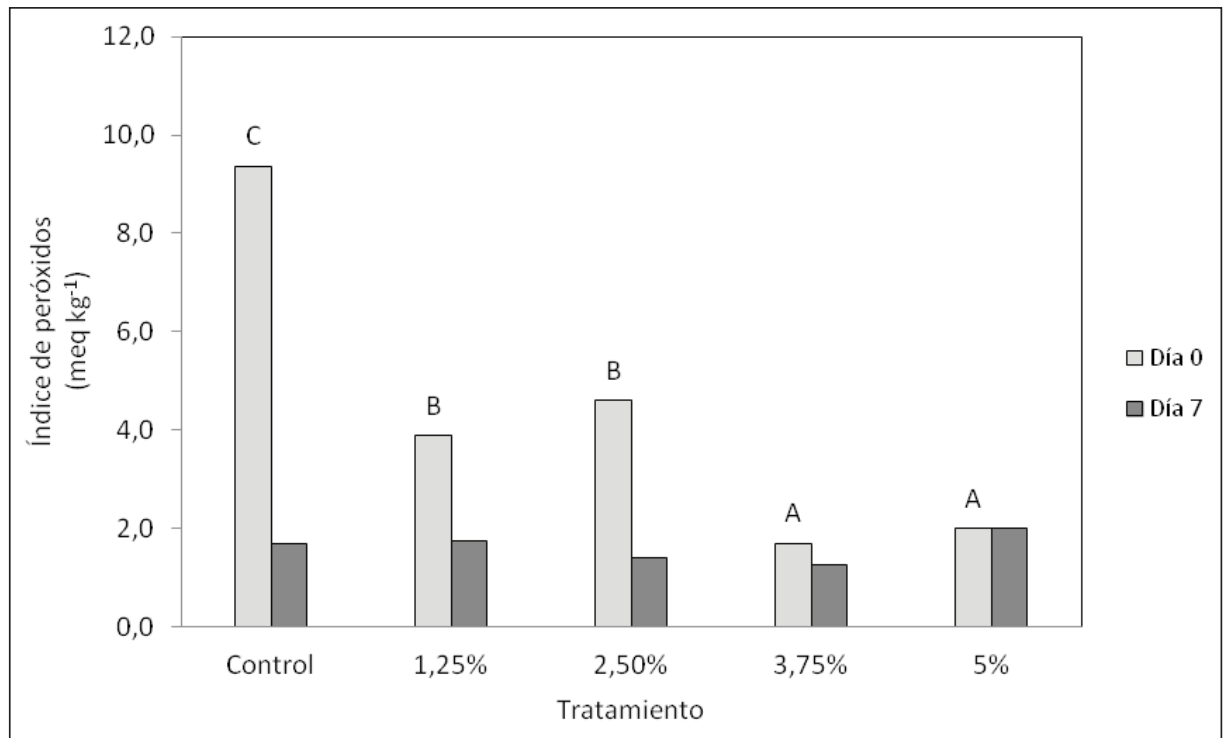

Fig. 1. Índice de peróxidos (meq $\mathrm{kg}^{-1}$ ) durante el almacenamiento de carne de muslo de pollos alimentados con dietas que incluyen orégano seco (Origanum vulgare L.).

Fig. 1. Peroxide index (meq $\mathrm{kg}^{-1}$ ) during the storage of meat from thigh muscles of broilers fed with diets containing dry oregano (Origanum vulgare L.).

Letras distintas indican diferencias entre tratamientos en cada tiempo según Duncan $(P \leq 0,05)$.

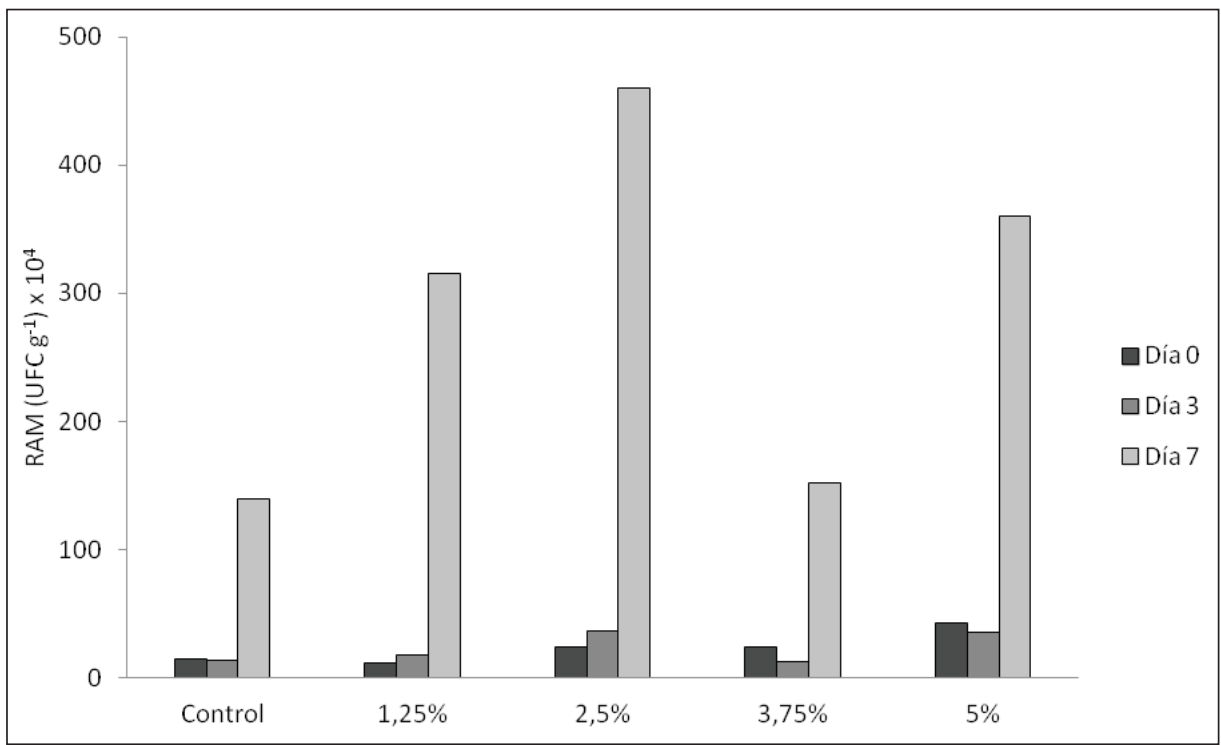

Fig. 2. Recuento total de microorganismos aerobios mesófilos (RAM) (UFC $\mathrm{g}^{-1} \mathbf{1 0}^{4}$ ) durante el almacenamiento de carne de pollos alimentados con dietas que incluyen orégano seco (Origanum vulgare L.).

Fig. 2. Total count of mesophilic aerobic microorganisms (TMA) (UFC $\mathrm{g}^{-1} \times 10^{4}$ ) during storage of poultry meat of broilers fed with diets containing dry oregano (Origanum vulgare L.).

hasta el día 7, donde aumentó considerablemente en todos los tratamientos. Sin embargo, el RAM determinado en este estudio está dentro del límite exigido por el Reglamento Sanitario de los Alimentos (RSA) (DS № 977/96. 1997), en el cual se exige un RAM máximo de $10^{7}$ en carne cruda. Nieto et al. (2010) también observaron un aumento del RAM durante la refrigeración de carne de ovejas alimentadas con dietas con sustitución de un 10 y $20 \%$ de su dieta basal con pellets fabrica- 
dos con $50 \%$ de cebada más $50 \%$ de romero, sin presentar diferencias significativas $(\mathrm{P}>0,05)$ en el RAM entre tratamientos, por lo tanto, no se obtuvo un efecto antimicrobiano en la carne pollo al incluir orégano seco en la dieta. Esto podría deberse a que los compuestos activos del orégano, el carvacrol y timol, que debieran conferir actividad antibacteriana y antifúngica, se transformarían durante el metabolismo en el hígado de animales monogástricos, conjugándose con ácido glucurónico $u$ otras sustancias endógenas del organismo, facilitando su excreción (Numpaque et al., 2011; García-García y Palou-García, 2008).

Por consiguiente, una forma más eficaz para controlar el crecimiento microbiano en la carne sería aplicarlo directamente sobre la carne de pollo o incluir el AE de orégano en envases activos, logrando así una concentración que permita la inhibición del crecimiento bacteriano (Velasco y Williams, 2011), como fue demostrado para hígado de pollo envasado adicionando EDTA, en atmósfera modificada, EDTA en atmósfera modificada, EDTA en atmósfera modificada más 0,1\% de aceite de orégano y EDTA en atmósfera modificada más $0,3 \%$ de aceite de orégano, pues los tratamientos que incluyeron aceite de orégano tu- vieron una vida útil, basado en el análisis sensorial, de 14 a 15 días, mientras que los tratamientos de atmósfera modificada y EDTA más atmósfera modificada solo de 7 a 9 días (Hasapidou y Savvaidis, 2011).

\section{Parámetros de color}

Los parámetros de color de la carne de pollo en los días 0, 3 y 7 de almacenamiento se muestran en las Tabla 2. El color de la carne depende principalmente del estado de oxidación de la mioglobina, la cual contiene hierro, si se oxida forma otro pigmento color marrón denominado metamioglobina (Faustman et al., 2010). En la carne el hierro se encuentra en la forma hemo, caracterizada por su mayor facilidad de absorción y biodisponibilidad, sin embargo, en la carne de pollo es menos abundante (Gil, 2010).

Los cortes de carne analizados en este estudio presentan diferencias de color, debido a que el contenido de mioglobina es superior en el muslo en comparación con el músculo pectoral (Cori et al., 2014). En la Tabla 2 se puede apreciar que en el músculo pectoral no hubo diferencias significativas $(\mathrm{P}>0,05)$ entre tratamientos en los parámetros de color al inicio del estudio (día 0). Sin embargo,

Tabla 2. Parámetros de color al día 0, 3 y 7 de almacenamiento en músculo pectoral y muslo de pollos alimentados con dietas que incluyen orégano seco (Origanum vulgare $\mathrm{L}$.).

Table 2. Color parameters at day 0,3 and 7 of storage of breast and thigh muscles from broilers fed with diets containing dry oregano (Origanum vulgare $\mathrm{L}$.$) .$

\begin{tabular}{|c|c|c|c|c|c|c|c|c|c|c|c|c|}
\hline & \multicolumn{6}{|c|}{ Parámetros de color. Músculo pectoral } & \multicolumn{6}{|c|}{ Parámetros de color. Muslo } \\
\hline & $\mathrm{L}^{*}$ & $a^{*}$ & $\mathbf{b}^{*}$ & $\mathrm{C}^{*}$ & $\mathrm{~h}^{*}$ & $\Delta \mathrm{E}$ & $\mathrm{L}^{*}$ & $a^{*}$ & $\mathbf{b}^{*}$ & $\mathrm{C}^{*}$ & $\mathrm{~h}^{*}$ & $\mathrm{DE}$ \\
\hline Tratamiento & \multicolumn{12}{|c|}{ Día 0} \\
\hline Control & $50,2 \mathrm{a}$ & $5,0 \mathrm{a}$ & $10,0 \mathrm{a}$ & $11,3 \mathrm{a}$ & $62,8 \mathrm{a}$ & - & $53,8 \mathrm{a}$ & $6,5 \mathrm{a}$ & $9,9 \mathrm{ab}$ & $11,9 \mathrm{ab}$ & $56,8 \mathrm{ab}$ & - \\
\hline $1,25 \%$ & $53,1 \mathrm{a}$ & $4,1 \mathrm{a}$ & $10,4 \mathrm{a}$ & 11,3 a & 68,8 a & 3,0 & $57,2 \mathrm{a}$ & $5,8 \mathrm{a}$ & $10,8 \mathrm{~b}$ & $12,3 \mathrm{ab}$ & $62,2 \mathrm{ab}$ & 3,4 \\
\hline $2,5 \%$ & $54,6 \mathrm{a}$ & $4,2 \mathrm{a}$ & $11,2 \mathrm{a}$ & $12,0 \mathrm{a}$ & $70,1 \mathrm{a}$ & 4,6 & $57,2 \mathrm{a}$ & $4,6 \mathrm{a}$ & $8,5 \mathrm{a}$ & $9.7 \mathrm{a}$ & $61,4 \mathrm{ab}$ & 4,1 \\
\hline $3,75 \%$ & $52,8 \mathrm{a}$ & $4,1 \mathrm{a}$ & $11,3 \mathrm{a}$ & $12,0 \mathrm{a}$ & $70,2 \mathrm{a}$ & 3,0 & $52,5 \mathrm{a}$ & $6,0 \mathrm{a}$ & $8,5 \mathrm{a}$ & $10,5 \mathrm{a}$ & 55,3 a & 1,8 \\
\hline \multirow[t]{2}{*}{$5 \%$} & $53,9 \mathrm{a}$ & $4,7 \mathrm{a}$ & $9,9 \mathrm{a}$ & $11,0 \mathrm{a}$ & $65,1 \mathrm{a}$ & 3,7 & $56,0 \mathrm{a}$ & $5,8 \mathrm{a}$ & $11,9 \mathrm{~b}$ & $13,3 \mathrm{~b}$ & $64,0 \mathrm{~b}$ & 1,8 \\
\hline & \multicolumn{12}{|c|}{ Día 3} \\
\hline Control & $56,2 \mathrm{a}$ & $4,9 \mathrm{a}$ & $10,5 \mathrm{a}$ & 11,7 a & $64,4 \mathrm{a}$ & - & $50,8 \mathrm{a}$ & $8,1 \mathrm{a}$ & $9,4 \mathrm{a}$ & $12,4 \mathrm{a}$ & 49,6 a & - \\
\hline $1,25 \%$ & $54,4 \mathrm{a}$ & $4,1 \mathrm{a}$ & $10,9 \mathrm{a}$ & 11,7 a & $69,7 \mathrm{a}$ & 2,0 & $51,3 \mathrm{a}$ & $6,9 a$ & $8,8 \mathrm{a}$ & $11,2 \mathrm{a}$ & $51,5 \mathrm{a}$ & 1,5 \\
\hline $2,5 \%$ & $55,7 \mathrm{a}$ & $4,5 \mathrm{a}$ & $11,3 \mathrm{a}$ & $12,2 \mathrm{a}$ & $68,3 \mathrm{a}$ & 1,0 & $54,9 \mathrm{a}$ & 6,1 a & $10,7 \mathrm{a}$ & $12,4 \mathrm{a}$ & $60,2 \mathrm{a}$ & 4,4 \\
\hline $3,75 \%$ & $56,8 \mathrm{a}$ & $4,0 \mathrm{a}$ & $11,0 \mathrm{a}$ & $11,8 \mathrm{a}$ & $70,6 \mathrm{a}$ & 1,2 & $51,9 \mathrm{a}$ & $7,3 \mathrm{a}$ & $9,8 \mathrm{a}$ & $12,3 \mathrm{a}$ & $52,9 \mathrm{a}$ & 1,2 \\
\hline \multirow[t]{2}{*}{$5 \%$} & $54,3 \mathrm{a}$ & $4,4 \mathrm{a}$ & $10,9 \mathrm{a}$ & 11,8 a & $68,1 \mathrm{a}$ & 2,0 & $53,1 \mathrm{a}$ & $7,7 \mathrm{a}$ & $9,9 \mathrm{a}$ & $12,6 \mathrm{a}$ & 51,7 a & 2,2 \\
\hline & \multicolumn{12}{|c|}{ Día 7} \\
\hline Control & $49,6 \mathrm{a}$ & $5,5 \mathrm{a}$ & $12,4 \mathrm{a}$ & $13,7 \mathrm{a}$ & $65,5 \mathrm{a}$ & - & $47,9 \mathrm{a}$ & $9,1 \mathrm{a}$ & $11,6 \mathrm{a}$ & $14,8 \mathrm{a}$ & 15,3 a & - \\
\hline $1,25 \%$ & $49,7 \mathrm{a}$ & $4,8 \mathrm{a}$ & $13,5 \mathrm{a}$ & 14,3 a & $70,3 \mathrm{a}$ & 1,3 & $50,5 \mathrm{a}$ & $7,9 \mathrm{a}$ & $11,7 \mathrm{a}$ & $14,2 \mathrm{a}$ & $14,9 \mathrm{a}$ & 2,8 \\
\hline $2,5 \%$ & $52,6 \mathrm{ab}$ & $4,4 \mathrm{a}$ & $13,2 \mathrm{a}$ & $13,9 \mathrm{a}$ & $71,7 \mathrm{a}$ & 3,3 & $53,0 \mathrm{a}$ & $6,3 \mathrm{a}$ & $11,4 \mathrm{a}$ & $13,1 \mathrm{a}$ & $15,1 \mathrm{a}$ & 5,8 \\
\hline $3,75 \%$ & $56,1 \mathrm{~b}$ & $3,5 \mathrm{a}$ & $12,3 \mathrm{a}$ & $12,8 \mathrm{a}$ & $74,2 \mathrm{a}$ & 6,8 & $50,7 \mathrm{a}$ & $7,2 \mathrm{a}$ & $10,7 \mathrm{a}$ & $12,9 \mathrm{a}$ & $14,3 \mathrm{a}$ & 3,5 \\
\hline $5 \%$ & $54,5 \mathrm{ab}$ & $3,7 \mathrm{a}$ & $11,6 \mathrm{a}$ & $12,2 \mathrm{a}$ & $72,7 \mathrm{a}$ & 5,3 & $52,8 \mathrm{a}$ & $7,7 \mathrm{a}$ & $12,5 \mathrm{a}$ & $14,7 \mathrm{a}$ & $13,4 \mathrm{a}$ & 5,0 \\
\hline
\end{tabular}

$\mathrm{L}^{*}$ : Luminosidad; $\mathrm{a}^{*}$ : Intensidad de rojo; $\mathrm{b}^{*}$ : Intensidad de amarillo; $\mathrm{C}^{*}$ : Croma; h: Ángulo de tono; $\Delta \mathrm{E}$ : Diferencia de color con respecto al tratamiento control.

Letras distintas indican diferencias significativas entre tratamientos según Duncan $(\mathrm{P} \leq 0,05)$. 
en muslos de pollo se presentaron diferencias significativas $(P \leq 0,05)$ entre tratamientos en el día 0 en el parámetro $b^{*}$ (intensidad de amarillo), encontrándose un mayor valor en el tratamiento de menor inclusión de orégano $(1,25 \%)$ y en el tratamiento de mayor inclusión de orégano (5\%). En el valor del croma y ángulo de tono también se presentaron diferencias significativas $(P \leq 0,05)$ entre tratamientos en el muslo al día 0 , presentando valores mayores el tratamiento de $5 \%$. Quiao et al. (2002) establecieron como parámetros de $\mathrm{L}^{*}$ para carne de ave normal, valores $48<\mathrm{L}^{*}<51$; carne pálida, blanda y exudativa, $\mathrm{L}^{*}>53$; y carne oscura, firme y seca, $\mathrm{L}^{*}<46$. En el presente ensayo los valores de $\mathrm{L}^{*}$ fueron mayores a 48 , lo cual corresponde a valores entre carne de ave normal y carne pálida, blanda y exudativa, sin diferencias significativas $(P \geq 0,05)$ entre tratamientos.

En el día 0 las diferencias de color $(\Delta \mathrm{E})$ con respecto al tratamiento control (C) estuvieron entre 1,8 y 4,6. Para que las diferencias de color sean perceptibles por el ojo humano, deben ser superiores a 5 ó $6 \Delta \mathrm{E}$. Sólo una persona bien preparada percibe diferencias de 3 ó $4 \Delta \mathrm{E}$ (LaCie, 2006). Por lo tanto, las diferencias obtenidas en este estudio, no serían perceptibles por los consumidores al inicio del almacenamiento. En el día 3 de almacenamiento en refrigeración no se observaron diferencias significativas $(\mathrm{P}>0,05)$ en el color entre tratamientos, y las diferencias de color $(\Delta \mathrm{E})$ fueron menores a 5 . El día 7 de almacenamiento en refrigeración en el músculo pectoral se presentaron diferencias significativas $(\mathrm{P} \leq 0,05)$ en la lu- minosidad $\mathrm{L}^{*}$, siendo mayor en el tratamiento de $3,75 \%$. Diferencias de color perceptibles con respecto al tratamiento control fueron obtenidas en el músculo pectoral de los tratamientos de mayor inclusión de orégano en la dieta (3,75 y 5\%). No se observaron diferencias significativas $(P>0,05)$ en el color del muslo entre tratamientos al día 7. Sin embargo, las diferencias de color con respecto al tratamiento control $(\Delta \mathrm{E})$ fueron mayores a 5 en los tratamientos 2,5 y $5 \%$. Du et al. (2000) encontró un efecto de la suplementación de ácido linoleico conjugado en la dieta de pollos en concentraciones de 1,$25 ; 2,5$; y $5 \%$ en los valores de $L^{*}$ de la carne después de 7 días de almacenamiento, siendo de 56,6; 55,6; y 53,2, respectivamente.

En el estudio realizado por Simitzis et al. (2010), los parámetros de color de carne de cerdos alimentados con suplemento dietético de AE de orégano en concentraciones de 0,$25 ; 0,5$; y $1 \mathrm{~mL}$ $\mathrm{kg}^{-1}$ no se vieron afectados significativamente. En estudios anteriores realizados en corderos y cabritos con inclusión de orégano seco en la dieta desde 1 a $5 \%$, tampoco se observaron diferencias significativas en los parámetros de color $\mathrm{L}^{*}, \mathrm{a}^{*} \mathrm{y}$ $\mathrm{b}^{*}$ de la carne cruda. Sin embargo, en dichos estudios no se evaluó el color de la carne durante el período de almacenamiento (Velasco et al., 2010; Velasco et al., 2011).

\section{Composición nutricional}

La composición nutricional de la carne de pollo del músculo pectoral y del muslo se presenta en la Tabla 3. El contenido de agua de la carne del

Tabla 3. Composición nutricional de carne del músculo pectoral y del muslo de pollos alimentados con dietas que incluyen orégano (Origanum vulgare L.) seco.

Table 3. Nutritional composition of breast and thigh muscles from broilers fed with diets containing dry oregano (Origanum vulgare L.).

\begin{tabular}{|c|c|c|c|c|}
\hline Tratamiento & Humedad & Proteínas & Lípidos & Minerales \%) \\
\hline \multicolumn{5}{|c|}{ 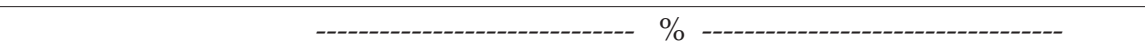 } \\
\hline \multicolumn{5}{|c|}{ Músculo pectoral } \\
\hline Control & 80,00 & 17,39 & 1,40 & 0,90 \\
\hline 1,25 & 84,00 & 15,54 & 1,48 & 1,20 \\
\hline $2,5 \%$ & 72,30 & 17,65 & 0,78 & 1,24 \\
\hline $3,75 \%$ & 70,60 & 15,61 & 2,38 & 1,28 \\
\hline $5 \%$ & 68,90 & 15,91 & 5,89 & 1,28 \\
\hline \multicolumn{5}{|c|}{ Muslo } \\
\hline Control & 70,00 & 16,45 & 1,47 & 1,43 \\
\hline 1,25 & 71,00 & 16,42 & 1,96 & 1,01 \\
\hline $2,5 \%$ & 70,70 & 16,97 & 1,55 & 1,37 \\
\hline $3,75 \%$ & 71,00 & 15,17 & 5,24 & 1,11 \\
\hline $5 \%$ & 69,00 & 14,76 & 3,65 & 1,16 \\
\hline
\end{tabular}

Valores expresados en base húmeda. 
músculo pectoral se encontró en el rango de 68,9 a $84 \%$, y en muslos entre 69 y $71 \%$; siendo similares a los valores obtenidos por Wattanachat et al. (2004) en pollos broilers. La carne de pollo tiene aproximadamente entre 16 y $25 \%$ de proteínas (Gil, 2010). En este estudio el contenido de proteínas del músculo pectoral osciló en el rango de 12,78 a $17,75 \%$, y en muslos entre 13,97 y $16,97 \%$, siendo menor en ambos casos a lo reportado por Wattanachat et al. (2004) con valores promedio de $20,59 \%$ en músculo pectoral y $19,08 \%$ en muslos. Según Gil (2010), el porcentaje de grasa o extracto etéreo (EE) debería encontrarse entre un 5-10\% en carnes magras y $10-30 \%$ en carnes grasas; por lo cual esta carne se puede clasificar como carne magra. El porcentaje de minerales ( $\mathrm{Se}, \mathrm{Zn}$, $\mathrm{Cu}, \mathrm{Mg}, \mathrm{Co}, \mathrm{P}, \mathrm{Cr}$, Ni y Fe en forma orgánica "hemo") determinado en las cenizas, concuerda con lo obtenido por Wattanachant et al. (2004) de aproximadamente $1 \%$. En un estudio realizado en carne de ovinos se observó un aumento en el extracto etéreo (contenido de lípidos) con suplementación de orégano en la dieta de los animales en un $1 \%$ comparado con el tratamiento control (Velasco et al., 2010). Esto indicaría una posible modificación del metabolismo de lípidos en los animales, lo cual requiere realizar otros estudios.

\section{Evaluación sensorial.}

En la Tabla 4 se presentan los resultados de la evaluación sensorial de la carne. Esta evaluación se realizó en el músculo pectoral (pechuga), ya que es un corte más homogéneo para ser presentado a los jueces. El color fue evaluado entre los valores 2-3 correspondiente a blanco amarillento y amarillo (Tabla 1). No se presentaron diferencias significativas $(P>0,05)$ en los parámetros de color entre los tratamientos con suplementación de orégano y el control. La intensidad del aroma del tratamiento control fue calificada con un valor 4, indicando una intensidad de aroma baja, y los tratamiento con suplementación de orégano fueron calificados con un valor de 3 , correspondiente a una intensidad de aroma moderada (Tabla 1). La aceptación general fue menor en el tratamiento control (Me disgusta) y mayor en los tratamientos con suplementación de orégano (Me es indiferente y Me gusta). Esto indica que la característica de intensidad de aroma podría afectar la aceptación de la carne, entre otras características sensoriales no evaluadas en este estudio, como jugosidad y textura. Hasapidou y Savvaidis (2011) al incorporar AE de orégano sobre la carne identificaron transferencia de sabores y olores desagradables en concentraciones de $0,1 \%$, razón por la cual recomiendan que su uso directo sea en concentraciones bajas. Estos resultados también concuerdan con Chouliara et al. (2007), quienes evaluaron el efecto combinado de atmósfera modificada y $1 \%$ o $0,1 \%$ de AE de orégano, obteniendo carne con mayor vida útil, pero de sabor y olor no aceptable en la concentración más alta.

\section{CONCLUSIONES}

La carne de pollos alimentados con dietas suplementadas con orégano seco presentó menor oxidación de lípidos al inicio del almacenamiento en refrigeración. Diferencias de color $(\Delta \mathrm{E})$ perceptibles por el ojo humano entre la carne de los tratamientos con mayor inclusión de orégano en la dieta $(2,5-5 \%)$ y el control se presentan a los siete días de almacenamiento. La suplementación de orégano seco en la dieta de pollos no tuvo un efecto antimicrobiano en la carne. La carne de pollos alimentados con suplementación de orégano seco $(1,25-5 \%)$ es aceptada por el consumidor y su mayor estabilidad al inicio del almacenamiento está dada por la menor oxidación de lípidos.

Tabla 4. Evaluación sensorial de color, intensidad de aroma y aceptación de carne de pollos del músculo pectoral, alimentados con dietas que incluyen orégano seco (Origanum vulgare L.).

Table 4. Sensory evaluation of color, aroma intensity and acceptance of breast muscle from broilers fed with diets containing dry oregano (Origanum vulgare L.).

\begin{tabular}{lccc}
\hline \multirow{2}{*}{ Tratamientos } & Color & Intensidad aroma & Aceptación \\
\cline { 2 - 4 } & \multicolumn{3}{c}{ Mediana } \\
\hline Control & $2,0 \mathrm{a}$ & $4,0 \mathrm{~b}$ & $2,0 \mathrm{a}$ \\
1,25 & $2,0 \mathrm{a}$ & $3,0 \mathrm{a}$ & $3,0 \mathrm{~b}$ \\
$2,5 \%$ & $2,0 \mathrm{a}$ & $3,0 \mathrm{a}$ & $3,0 \mathrm{~b}$ \\
$3,75 \%$ & $2,5 \mathrm{a}$ & $3,0 \mathrm{a}$ & $4,0 \mathrm{~b}$ \\
$5 \%$ & $2,0 \mathrm{a}$ & $3,0 \mathrm{a}$ & $3,5 \mathrm{~b}$ \\
\hline
\end{tabular}

Letras distintas indican diferencias entre tratamientos según Kruskal-Wallis $(P \leq 0,05)$. 


\section{LITERATURA CITADA}

AOAC. 1997. Official methods of analysis. AOAC International. Volume II. (16th. ed.). AOAC International, Gaithersburg, USA.

Balzarini, M.G., L. González, M. Tablada, F. Casanoves, J.A. Di Rienzo y C.W. Robledo. 2008. Infostat. Manual del usuario. Editorial Brujas, Córdova, Argentina.

Bampidis, V.A., V. Christodoulou, P. Florou-Paneri, E. Christaki, A.B. Spais, and P.S. Chatzopoulou. 2005. Effect of dietary dried oregano leaves supplementation on performance and carcass characteristics of growing lambs. Animal Feed Science and Technology 121:285-295.

Bonfanti, C., R. Iannì, A. Mazzaglia, C.M. Lanza, E.M. Napoli, and G. Ruberto. 2012. Emerging cultivation of oregano in Sicily: Sensory evaluation of plants and chemical composition of essential oils. Ind. Crop. Prod. 35:160-165.

Botsoglou, N.A., D.J. Fletouris, P. Florou-Paneri, E. Christaki, and A.B. Spais. 2003a. Inhibition of lipid oxidation in long-term frozen stored chicken meat by dietary oregano essential oil and $\alpha$-tocopheryl acetate supplementation. Food Research Int. 36:207-213.

Botsoglou, N.A., E. Christaki, D.J. Fletouris, P. Florou-Paneri, and A.B. Spais. 2002. The effect of dietary oregano essential oil on lipid oxidation in raw and cooked chicken during refrigerated storage. Meat Sci. 62(2):259-265.

Botsoglou, N.A., S.H. Grigoropoulou, E. Botsoglou, A. Govaris, and G. Papageorgiou. $2003 \mathrm{~b}$. The effects of dietary oregano essential oil and $\alpha$-tocopheryl acetate on lipid oxidation in raw and cooked turkey during refrigerated storage. Meat Sci. 65:1193-1200.

Burt, S. 2004. Essential oils: their antibacterial properties and potential applications in foods-a review. Int. J. Food Microbiol. 94(3):223-253.

Chaijan, M. 2008. Review: Lipid and myoglobin oxidations in muscle foods. Songklanakarin J. Sci. Technol. 30(1):47-53.

Chouliara, E., A. Karatapanis, I.N. Savvaidis, and M.G. Kontominas. 2007. Combined effect of oregano essential oil and modified atmosphere packaging on shelf-life extension of fresh chicken breast meat, stored at $4^{\circ} \mathrm{C}$. Food Microbiology 24(6):607-617.

Cori, M.E., C. Michelangeli, V. De Basilio, R. Figueroa, y N. Rivas. 2014. Solubilidad proteica, contenido de mioglobina, color y $\mathrm{pH}$ de la carne de pollo, gallina y codorniz. Arch. Zootec. 63(241):133-143.
De Falco, E., G. Roscigno, S. Landolfi, E. Scandolera, and F. Senatore. 2014. Growth, essential oil characterization, and antimicrobial activity of three wild biotypes of oregano under cultivation condition in Southern Italy. Ind. Crop Prod. 62:242-249.

Diario Oficial de la República de Chile. 1997. Decreto Supremo № 977/96. 1997. Reglamento sanitario de los alimentos. 13 mayo 1997. Santiago, Chile.

Dorman, H.J., and S.G. Deans. 2000. Antimicrobial agents from plants: antibacterial activity of plant volatile oils. J. Appl. Microbiol. 88(2):308-316.

Du, M., D.U. Ahn, K.C. Nam, and J.L. Sell. 2000. Influence of dietary conjugated linoleic acid on volatile profiles, color and lipid oxidation of irradiated raw chicken meat. Meat Sci. 56(4):387-395.

Elgayyar, M., F.A. Draughon, D.A. Golden, and J.R. Mount. 2001. Antimicrobial activity of essential oils from plants against selected pathogenic and saprophytic microorganisms. J. Food Protect. 64:1019-1024.

Fasseas, M.K., K.C. Mountzouris, P.A. Tarantilis, M. Polissiou, and G. Zervas. 2007. Antioxidant activity in meat treated with oregano and sage essential oils. Food Chemist. 106:1188-1194.

Faustman C., Q. Sun, R. Mancini, and S. Suman. 2010. Myoglobin and lipid oxidation interactions: Mechanistic bases and control. Meat Sci. 86:86-94.

Friedman, M., P.R. Henika, and R.E. Mandrell. 2002. Bactericidal activities of plant essential oils and some of their isolated constituents against Campylobacter jejuni, Escherichia coli, Listeria monocytogenes, and Salmonella enterica. J. Food Prot. 65(10):1545-1560.

García-García R.M., y E. Palou-García. 2008. Mecanismos de acción antimicrobiana de timol y carvacrol sobre microorganismos de interés en alimentos. Temas Selectos de Ingeniería de Alimentos 2-2:41-51.

Gil, A. 2010. Tratado de nutrición: composición y calidad nutritiva de los alimentos. Tomo II. (2a. ed.). Médica Panamericana, Madrid, España.

Hasapidou, A., and I.N. Savvaidis. 2011. The effects of modified atmosphere packaging, EDTA and oregano oil on the quality of chicken liver meat. Food Research International 44:2751-2756.

Hashim, I.B., A.S. Hussein, and H.S. Afifi. 2013. Quality of breast and thigh meats when broilers are fed rations containing graded levels of sugar syrup. Poult. Sci 92:2195-2200. 
Hunter Associates Laboratory. 2008. CIE L*C"h color scale [on line]. HunterLab Aplications Note. 8(11):1-4. Available at <https://support. hunterlab.com/hc/en-us/articles/203285709CIE-L-C-h-Color-Scale-an09-96a>. (Accessed 16 November 2012)

Huyghebaert, G., R. Ducatelle, F., and Van Immerseel. 2011. An update on alternatives to antimicrobial growth promoters for broilers. The Vet J. 187:182-188.

LaCie. 2006. Libro blanco de la gestión del color. 3. Espacios de color y conversión de colores. Disponible en <http://www.prodesgrafica. com/userfiles/file/LACIE\%20colormanagement_es.pdf $>$ (Consultado 21 enero 2016).

Lambert, R.J.W., P.N. Skandamis, P.J. Coote, and G.J.E. Nychas. 2001. A study of the minimum inhibitory concentration and mode of action of oregano essential oil, thymol and carvacrol. Journal of Applied Microbiology 91:453-462.

Lv, F., H. Liang, Q. Yuan, and C. Li. 2011. In vitro antimicrobial effects and mechanism of action of selected plant essential oil combinations against four food-related microorganisms. Food Res. Int. 44:3057-3064.

Marino, M., C. Bersani, and G. Comi, 2001. Impedance measurements to study the antimicrobial activity of essential oils from Lamiaceae and Compositae. Int. J. Food Microbiol. 67(3):187-195.

Mc Dermott, P.F., S. Zhao, D.D. Wagner, S. Simjee, R.D. Walker, and D.G. White. 2002. The food safety perspective of antibiotic resistance. Anim. Biotechnol. 13(1):71-84.

Mohamed, H.M.H., and H.A. Mansour. 2012. Incorporating essential oils of marjoram and rosemary in the formulation of beef patties manufactured with mechanically deboned poultry meat to improve the lipid stability and sensory attributes. LWT Food Sci. Tech. 45:79-87.

Nieto, G., P. Díaz, S. Bañón, and M. Garrido. 2010. Dietary administration of ewe diets with a distillate from rosemary leaves (Rosmarinus officinalis L.): influence on lamb meat quality. Meat Sci. 84(1):23-29.

NRC. 1994. Nutrient requirements of poultry. (9th. ed.). National Academy Press. National Research Council, Washington D.C., USA.

Numpaque M., L.A. Oviedo, J. H. Gil, C.M. García, and D. Durango. 2011. Thymol and carvacrol: biotransformation and antifungal activity against the plant pathogenic fungi Colletotrichum acutatum and Botryodiplodia theobromae. Tropical Plant Pathology 36(1): 003-013.
Okubo, T., Y. Yokoyama, K. Kano, and I. Kano. 2003. Cell death induced by the phenolic antioxidant tert-butylhydroquinone and its metabolite tert-butylquinone in human monocytic leukemia U937 cells. Food Chemical Toxicol. 41:679-688.

Oral, N. L. Vatansever, Ç. Sezer, B. Aydın, A. Güven, M. Gülmez, et al. 2009. Effect of absorbent pads containing oregano essential oil on the shelf life extension of overwrap packed chicken drumsticks stored at four degrees Celsius. Poultry Sci. 88:1459-1465.

Pearson, D. 1986. Técnicas de laboratorio para análisis de alimentos. Acribia, Zaragoza, España.

Quiao, M. D. L. Fletcher, J. K. Northcutt, and D. P. Smith. 2002. The relationship between raw broiler breast meat color and composition. Poultry Sci. 81:422-427.

Quinn, P., y B. Markey. 2005. Elementos de microbiología veterinaria. Acribia, Zaragoza, España.

Richards M. P., A. M. Modra, and R. Li. 2002. Role of deoxyhemoglobin in lipid oxidation of washed cod muscle mediated by trout, poultry and beef hemoglobins. Meat Sci. 62:157-163.

Rodríguez, E. A., A. Arias, E. Vásquez, J. Martínez, E. Stashenko. 2012. Rendimiento y capacidad antioxidante de extractos de Rosmarinus officinalis, Salvia officinalis y Psidium guajava obtenidos con $\mathrm{CO}_{2}$ supercrítico. Rev. Acad. Colomb. Cienc. Exact. Fis. Nat. [online]. 36(140):305-316. Available from <http:// www.scielo.org.co/scielo.php?script $=$ sci arttext\&pid=S0370-39082012000300001\&1ng=en\&nrm=iso>. ISSN 0370-3908 (Consulta 18 mayo 2016).

Simitzis P.E., S.G. Deligeorgis, J.A. Bizelis, A. Dardamani, I. Theodosiou, and K. Fegeros. 2008. Effect of dietary oregano oil supplementation on lamb meat characteristics. Meat Sci. 79(2):217-223

Simitzis, P.E., G.K. Symeon, M.A. Charismiadou, J.A. Bizelis, and S.G. Deligeorgis. 2010. The effects of dietary oregano oil supplementation on pig meat characteristics. Meat Sci. 84(4):670-676.

Solomakos, N., A. Govaris, P. Koidis, and N. Botsoglou. 2008. The antimicrobial effect of thyme essential oil, nisin, and their combination against Listeria monocytogenes in minced beef during refrigerated storage. Food Microbiol. 25:120-127.

Velasco, V., and P. Williams. 2011. Improving meat quality through natural antioxidants. Chilean J. Agricultural Research 71(2):313322. 
Velasco, V., C. Orellana, P. Williams, J. Campos, y P. Melín. 2011. Parámetros de calidad de carne de caprinos alimentados con dieta suplementada con orégano (Origanum vulgare L.) seco. Agro-Ciencia 27(2):95-104.

Velasco, V., J. Parada, P. Williams, J. Campos, y P. Melín. 2010. Estudio preliminar de calidad de carne de ovinos alimentados con dieta suplementada con orégano seco. Agro-Ciencia 26(1):45-53.

Wattanachant, S., S. Benjakul, and D.A. Ledward. 2004. Composition, color and texture of thai indigenous and broiler chicken muscles. Poult. Sci. 83(1):123-128.

Wittig de Penna, E. 2001. Evaluación sensorial: una metodología actual para tecnología de alimentos. Universidad de Chile. Disponible en <http://mazinger.sisib.uchile.cl/repositorio/lb/ciencias_quimicas_y_farmaceuticas/ wittinge01/index.html> (Consulta 18 agosto 2011). 